\title{
The Test of Entanglement of Polarization States of a Semi-Classical Optical Parametric Oscillator
}

\author{
Gerald Mugaya Lisamadi ${ }^{1}$, Boniface Otieno Ndinya ${ }^{2}$, Joseph Akeyo Omolo ${ }^{1}$ \\ ${ }^{1}$ Department of Physics and Materials Science, Maseno University, Maseno, Kenya \\ ${ }^{2}$ Department of Physics, Masinde Muliro University of Science and Technology, Kakamega, Kenya
}

Email address:

geraldmugaya@gmail.com (G. M. Lisamadi), bonifacendinya@gmail.com (B. O. Ndinya), ojakeyo04@yahoo.co.uk (J. A. Omolo)

\section{To cite this article:}

Gerald Mugaya Lisamadi, Boniface Otieno Ndinya, Joseph Akeyo Omolo. The Test of Entanglement of Polarization States of a SemiClassical Optical Parametric Oscillator. American Journal of Modern Physics. Vol. 6, No. 3, 2017, pp. 37-42. doi: 10.11648 j.ajmp.20170603.11

Received: March 29, 2017; Accepted: April 10, 2017; Published: May 8, 2017

\begin{abstract}
We study the dynamical continuous variable entanglement in a semi-classical Optical parametric oscillator (OPO). The general time evolving photon polarization state vectors arising from exact analytical solutions of Heisenberg's equations of the system are used to obtain the photon polarization Bell state vectors. The reduced density matrices of photon polarization Bell state vectors of the semi-classical OPO produce a greater violation of CHSH Bell's inequality beyond the Cirel'son's inequality.
\end{abstract}

Keywords: Optical Parametric Oscillator, Bell State Vectors, Reduced Density Matrices, Entanglement

\section{Introduction}

Quantum entanglement is a physical phenomenon that occurs when pairs or groups of particles are generated or interact in such a manner that the quantum state of each particle cannot be described independently; instead, a quantum state is given for the system as a whole. Entanglement has found many applications in the fields of quantum computation and quantum information processing such as quantum cryptography, quantum dense coding, entanglement swapping, quantum lithography and quantum teleportation [1]. Quantum teleportation (transfer of quantum states between distant locations without an intervening medium) has been achieved over long distances [2, 3, 4]. On August 16, 2016, China launched the world's first "quantum satellite", a communication system incapable of being hacked and stretching over a distance of $2000 \mathrm{~km}$ [5].

Continuous variable entanglement was demonstrated for the first time in the Optical parametric oscillator (OPO) operating below threshold in 1990. Entanglement in the above-threshold OPO remained an experimental challenge until 2005, when it was first observed by Villar S. and Cassemiro N. (2005) [6]. Entanglement features for a full quantum treatment of OPO has been studied where time evolution equations are solved through writing of the density operator equation in the Wigner representation using equivalent Langevin equations to obtain analytical results [7]. $\mathrm{Su}$ and Tan (2006) [8] observed a two-color entanglement measured to the above-threshold OPO only. The multipartite nature of entanglement was verified by evaluating the van Loock-Furusawa criterion for a particular set of entanglement witnesses deduced from physical considerations [9]. Johansson R. (2014) [10] investigated theoretically the conditions under which a multi-mode nano-mechanical resonator, operating as a purely mechanical parametric oscillator, can be driven into highly non-classical states. Quantum entangled states of the system violate Bell inequalities with homodyne quadrature measurements. Chakrabarti R. and Jenisha J. (2015) [11] studied the evolution of a bipartite entangled quasi-bell state in a strongly coupled qubit oscillator system in the presence of a static bias, and extended it to the ultra-strong coupling regime. Adiabatic approximation was used to obtain reduced density matrix of the qubit for the strong coupling domain in closed form involving linear combinations of the Jacobi theta functions. Apart from employing the adiabatic approximation no other simplification has been made for deriving the reduced density matrix elements. A test of Bell inequality using polarization entangled photons from a Spontaneous 
Parametric down Conversion (SPDC) has shown a violation of Bell's inequality [12]. In the current paper, we use reduced density matrices of polarization states of semi-classical OPO to test the violation of CHSH Bell's inequality, under all conditions of interaction.

The paper is organized as follows. In section 2, the Hamiltonian of the system is developed and used to obtain the time evolution operator of the system. In section 3, the time evolving photon polarization Bell states are constructed. In section 4, the test of entanglement of polarization states of OPO using reduced density matrices and Bell inequalities is presented. A conclusion is provided in section 5.

\section{The Time Evolution Operator}

The OPO is an alternative tool for non-linear generation of entangled photons where a pump photon is converted into two lower energy beams, known as the signal photon and the idler photon occurring due to excitation of a material medium when struck by an external electromotive force. The signal photon is taken to be initially in the horizontal polarization state and the idler photon is taken to be initially in the vertical polarization state. The photon is therefore in a superposition of horizontal and vertical polarization. The horizontal polarization state vector represents the basic unit vector $|0\rangle$ and the vertical polarization state vector represents the basic unit vector $|1\rangle$ according to the definition

$$
|H\rangle=|0\rangle=\left(\begin{array}{l}
1 \\
0
\end{array}\right) \quad, \quad|V\rangle=|1\rangle=\left(\begin{array}{l}
0 \\
1
\end{array}\right)
$$

The two-mode Hamiltonian of semi-classical OPO is described by [13]

$$
H=\hbar\left(\omega_{a} \hat{a}^{+} \hat{a}+\omega_{b} \hat{b}^{+} \hat{b}+\alpha \hat{a}^{+} \hat{b}+\alpha^{*} \hat{b}^{+} \hat{a}\right)
$$

where $\hat{a}\left(\hat{a}^{+}\right)$is the signal annihilation (creation) operators, $\omega_{a}$ is the signal frequency, $\hat{b}\left(\hat{b}^{+}\right)$is the idler annihilation (creation) operators, $\omega_{b}$ is the idler frequency and $\alpha\left(\alpha^{*}\right)$ is a time-dependent interaction parameter which varies harmonically with time in the form of a rotating pump field of frequency $\left(\omega_{p}\right)$, according to

$$
\alpha(t)=\alpha e^{-i \omega_{p} t} \quad ; \quad \alpha^{*}(t)=\alpha e^{i \omega_{p} t} \quad ; \quad \alpha=|\alpha(t)|=\text { constant }
$$

The dynamics of the semi-classical OPO is described through Heisenberg's equations

$$
\begin{gathered}
i \hbar \frac{d \hat{a}}{d t}=[\hat{a}, \hat{\mathrm{H}}]=\frac{\partial \hat{\mathrm{H}}}{\partial \hat{a}^{+}}=\hbar\left(\omega_{a} \hat{a}+\alpha(t) \hat{b}\right) \\
i \hbar \frac{d \hat{b}}{d t}=[\hat{b}, \hat{\mathrm{H}}]=\frac{\partial \hat{\mathrm{H}}}{\partial \hat{b}^{+}}=\hbar\left(\alpha^{*}(t) \hat{a}+\omega_{b} \hat{b}\right)
\end{gathered}
$$

Equations (3a) and ( $3 b)$ can be expressed in matrix form as

$$
i \hbar \frac{d \hat{A}}{d t}=\hbar\left(\begin{array}{cc}
\omega_{a} & \alpha(t) \\
\alpha^{*}(t) & \omega_{b}
\end{array}\right) \hat{A}
$$

where $\hat{A}$ is the two-component photon pair polarization vector defined as

$$
\hat{A}=\left(\begin{array}{l}
\hat{a} \\
\hat{b}
\end{array}\right)=\hat{a}|0\rangle+\hat{b}|1\rangle=\hat{a}|H\rangle+\hat{b}|V\rangle
$$

Equation (4a) can be expressed in alternative form as

$$
i \hbar \frac{d \hat{A}}{d t}=\hbar\left(\Omega_{a b} \hat{S}_{0}+\omega_{a b} \hat{S}_{z}+\alpha(t) \hat{S}_{+}+\alpha^{*}(t) \hat{S}_{-}\right) \hat{A}
$$

where

$$
\Omega_{a b}=\omega_{a}+\omega_{b} \quad ; \quad \omega_{a b}=\omega_{a}-\omega_{b},
$$

$$
\hat{S}_{0}=\frac{1}{2}\left(\begin{array}{cc}
1 & 0 \\
0 & 1
\end{array}\right) \quad ; \quad \hat{S}_{z}=\frac{1}{2} \sigma_{z}=\frac{1}{2}\left(\begin{array}{cc}
1 & 0 \\
0 & -1
\end{array}\right) \text {, }
$$

$$
\hat{S}_{+}=\frac{1}{2}\left(\sigma_{x}+i \sigma_{y}\right)=\left(\begin{array}{ll}
0 & 1 \\
0 & 0
\end{array}\right) \quad, \quad \hat{S}_{-}=\frac{1}{2}\left(\sigma_{x}-i \sigma_{y}\right)=\left(\begin{array}{ll}
0 & 0 \\
1 & 0
\end{array}\right)
$$

and $\sigma_{i} ; i=x, y, z$ are the Pauli spin operators. Equation (5a) is solved to obtain the general form of time evolution photon polarization vector, expressed as

$$
\hat{A}(t)=U_{A}(t) \hat{A}(0)
$$

where $U_{A}(t)$ is the time evolution operator,

$$
U_{A}(t)=e^{-i \Omega} a b^{\hat{S}_{0} t} e^{-i \omega_{p} t \hat{S}_{z}} e^{-i t\left(\Delta \hat{S}_{z}+\alpha(t) e^{i \omega_{p} t} \hat{S}_{+}+\alpha^{*}(t) e^{-i \omega_{p} t} \hat{S}_{-}\right)}
$$

and $\Delta$ is a constant detuning parameter,

$$
\Delta=\omega_{a b}-\omega_{p}
$$

We substitute for spin operators $\hat{S}_{0}, \hat{S}_{z}, \hat{S}_{+}$and $\hat{S}_{-}$(5c-d) in (6b) to obtain the final matrix as

$$
U_{A}(t)=\left(\begin{array}{cc}
\mu(t) e^{-\frac{i}{2}\left(\Omega_{a b}+\omega_{p}\right) t} & v(t) e^{-\frac{i}{2}\left(\Omega_{a b}+\omega_{p}\right) t} \\
-v^{*}(t) e^{-\frac{i}{2}\left(\Omega_{a b}-\omega_{p}\right) t} & \mu^{*}(t) e^{-\frac{i}{2}\left(\Omega_{a b}-\omega_{p}\right) t}
\end{array}\right)
$$

where 


$$
\begin{gathered}
\mu(t)=\cos (\beta t)-\frac{i \Delta}{2 \beta} \sin (\beta t) \\
v(t)=-\frac{i \alpha(t) e^{i \omega_{p} t}}{\beta} \sin (\beta t) \\
\beta=|\alpha| \sqrt{1+k^{2}} \quad ; \quad k=\frac{\Delta}{2|\alpha|}
\end{gathered}
$$

Similarly, if we consider the signal photon represented by mode 'a' to be initially in the vertical polarization state $|V\rangle$ and the idler photon represented by mode ' $\mathrm{b}$ ' to be initially in the horizontal polarization state $|H\rangle$, and follow steps given by equations (2a) to (7d), we obtain the alternative final form of time evolution operator as

$$
U_{B}(t)=\left(\begin{array}{ll}
\mu^{*}(t) e^{-\frac{i}{2}\left(\Omega_{a b}-\omega_{p}\right) t} & -v^{*}(t) e^{-\frac{i}{2}\left(\Omega_{a b}-\omega_{p}\right) t} \\
v(t) e^{-\frac{i}{2}\left(\Omega_{a b}+\omega_{p}\right) t} & \mu(t) e^{-\frac{i}{2}\left(\Omega_{a b}+\omega_{p}\right) t}
\end{array}\right)
$$

\section{Bell State Vectors}

For a two-particle system where the signal photon is denoted by mode ' $a$ ' is taken to be initially in the horizontal polarization state and the idler photon is denoted by mode ' $\mathrm{b}$ ' is taken to be initially in the vertical polarization state or vice versa. Then the maximum entangled Bell state vector of the photon is the superposition of horizontal and vertical polarization presented in simple form as [14]

$$
\begin{array}{r}
\left|\phi_{ \pm}\right\rangle_{a b}=\frac{1}{\sqrt{2}}\left(|0\rangle_{a}|0\rangle_{b} \pm|1\rangle_{a}|1\rangle_{b}\right)=\frac{1}{\sqrt{2}}\left(|H\rangle_{a}|H\rangle_{b} \pm|V\rangle_{a}|V\rangle_{b}\right) \\
\left|\psi_{ \pm}\right\rangle_{a b}=\frac{1}{\sqrt{2}}\left(|0\rangle_{a}|1\rangle_{b} \pm|1\rangle_{a}|0\rangle_{b}\right)=\frac{1}{\sqrt{2}}\left(|H\rangle_{a}|V\rangle_{b} \pm|V\rangle_{a}|H\rangle_{b}\right)
\end{array}
$$

Using equations (1), (7a) and (8) the four possible eigen states of the two-particle system in (9a) and (9b) takes the form

$$
\begin{gathered}
|H\rangle_{a}=U_{A}(t)|H\rangle=\left(\mu(t) e^{-\frac{i}{2}\left(\Omega_{a b}+\omega_{p}\right) t}|0\rangle-v^{*}(t) e^{-\frac{i}{2}\left(\Omega_{a b}-\omega_{p}\right) t}|1\rangle\right) \\
|H\rangle_{b}=U_{B}(t)|H\rangle=\left(\mu^{*}(t) e^{-\frac{i}{2}\left(\Omega_{a b}-\omega_{p}\right) t}|0\rangle+v(t) e^{-\frac{i}{2}\left(\Omega_{a b}+\omega_{p}\right) t}|1\rangle\right) \\
|V\rangle_{a}=U_{B}(t)|V\rangle=\left(-v^{*}(t) e^{-\frac{i}{2}\left(\Omega_{a b}-\omega_{p}\right) t}|0\rangle+\mu(t) e^{-\frac{i}{2}\left(\Omega_{a b}+\omega_{p}\right) t}|1\rangle\right) \\
|V\rangle_{b}=U_{B}(t)|V\rangle=\left(v(t) e^{-\frac{i}{2}\left(\Omega_{a b}+\omega_{p}\right) t}|0\rangle+\mu^{*}(t) e^{-\frac{i}{2}\left(\Omega_{a b}-\omega_{p}\right) t}|1\rangle\right)
\end{gathered}
$$

Using the general time evolving photon polarization state vector equations $(10 a-d)$ in $(9 a)$ and $(9 b)$, the four time evolution photon polarization Bell state vector reduces to

$$
\begin{aligned}
& \left|\phi^{+}\right\rangle=e^{-i \Omega_{a b} t}\left(\begin{array}{c}
\cos ^{2}(\beta t)-\left(\frac{\Delta^{2}+4|\alpha|^{2}}{4 \beta^{2}}\right) \sin ^{2}(\beta t) \\
-\frac{i \alpha}{\beta} \cos \left(\omega_{p} t\right) \sin (2 \beta t)+\frac{i \Delta \alpha}{\beta^{2}} \sin \left(\omega_{p} t\right) \sin ^{2}(\beta t) \\
-\frac{i \alpha}{\beta} \cos \left(\omega_{p} t\right) \sin (2 \beta t)+\frac{i \Delta \alpha}{\beta^{2}} \sin \left(\omega_{p} t\right) \sin ^{2}(\beta t) \\
\cos ^{2}(\beta t)-\left(\frac{\Delta^{2}+4|\alpha|^{2}}{4 \beta^{2}}\right) \sin ^{2}(\beta t)
\end{array}\right) \\
& \left|\phi^{-}\right\rangle=e^{-i \Omega_{a b} t}\left(\begin{array}{c}
\cos ^{2}(\beta t)-\left(\frac{\Delta^{2}-4|\alpha|^{2}}{4 \beta^{2}}\right) \sin ^{2}(\beta t) \\
-\frac{\alpha}{\beta} \sin \left(\omega_{p} t\right) \sin (2 \beta t)-\frac{\Delta \alpha}{\beta^{2}} \cos \left(\omega_{p} t\right) \sin ^{2}(\beta t) \\
\frac{\alpha}{\beta} \sin \left(\omega_{p} t\right) \sin (2 \beta t)+\frac{\Delta \alpha}{\beta^{2}} \cos \left(\omega_{p} t\right) \sin ^{2}(\beta t) \\
-\cos ^{2}(\beta t)+\left(\frac{\Delta^{2}-4|\alpha(t)|^{2}}{4 \beta^{2}}\right) \sin ^{2}(\beta t)
\end{array}\right) \\
& \left|\psi^{+}\right\rangle=e^{-i \Omega_{a b} t}\left(\begin{array}{c}
-\frac{i \alpha}{\beta} \cos \left(\omega_{p} t\right) \sin (2 \beta t)+\frac{i \Delta \alpha}{\beta^{2}} \sin \left(\omega_{p} t\right) \sin ^{2}(\beta t) \\
\cos ^{2}(\beta t)-\left(\frac{\Delta^{2}+4|\alpha|^{2}}{4 \beta^{2}}\right) \sin ^{2}(\beta t) \\
\cos ^{2}(\beta t)-\left(\frac{\Delta^{2}+4|\alpha|^{2}}{4 \beta^{2}}\right) \sin ^{2}(\beta t) \\
-\frac{i \alpha}{\beta} \cos \left(\omega_{p} t\right) \sin (2 \beta t)+\frac{i \Delta \alpha}{\beta^{2}} \sin \left(\omega_{p} t\right) \sin ^{2}(\beta t)
\end{array}\right) \\
& \left|\psi^{-}\right\rangle=e^{-i \Omega_{a b} t}\left(\begin{array}{c}
-\frac{i \alpha}{\beta} \sin \left(\omega_{p} t\right) \sin (2 \beta t)-\frac{\Delta \alpha}{\beta^{2}} \cos \left(\omega_{p} t\right) \sin ^{2}(\beta t) \\
\cos ^{2}(\beta t)-\left(\frac{\Delta^{2}-4|\alpha|^{2}}{4 \beta^{2}}\right) \sin ^{2}(\beta t) \\
-\cos ^{2}(\beta t)+\left(\frac{\Delta^{2}-4|\alpha(t)|^{2}}{4 \beta^{2}}\right) \sin ^{2}(\beta t) \\
\frac{\alpha}{\beta} \sin \left(\omega_{p} t\right) \sin (2 \beta t)+\frac{\Delta \alpha}{\beta^{2}} \cos \left(\omega_{p} t\right) \sin ^{2}(\beta t)
\end{array}\right)
\end{aligned}
$$

\section{Reduced Density of State}

The Clauser-Horne-Shimony-Holt (CHSH)

Bell inequality defined as

$$
-2 \leq S \leq+2
$$

is commonly used to test the nature of entanglement of photon polarization states[15]. In (12) $S$ represents the reduced density matrix for Bell state vectors [16]. Entanglement is exhibited by violations of the Bell inequality. The larger the violation of the Bell inequality the more the entanglement present in the system.

We determine reduced density matrices by obtaining the trace of density matrices under the following conditions of interaction: 


\subsection{Resonance}

At resonance,

$$
|\alpha|>>\Delta
$$

and $(7 \mathrm{~d})$ reduces to

$$
\beta=|\alpha| \quad ; \quad k=0
$$

We use the condition in (13a) and (13b) in polarization Bell state vectors (11a-d) to obtain the trace of the density matrices under resonance condition as

$$
\begin{gathered}
\operatorname{Tr} \hat{\rho}_{a b_{+}}^{a}(t)=\operatorname{Tr}_{b}\left(\left|\phi_{+}\right\rangle\left\langle\phi_{+}\right|\right)=2-2 \sin ^{2}\left(\omega_{p} t\right) \sin ^{2}(2|\alpha| t) \\
\operatorname{Tr} \hat{\rho}_{a b_{-}}^{a}(t)=\operatorname{Tr}_{b}\left(\left|\phi_{-}\right\rangle\left\langle\phi_{-}\right|\right)=2+2 \sin ^{2}\left(\omega_{p} t\right) \sin ^{2}(2|\alpha| t) \\
\operatorname{Tr} \hat{\rho}_{b a_{+}}^{b}(t)=\operatorname{Tr}_{a}\left(\left|\psi_{+}\right\rangle\left\langle\psi_{+}\right|\right)=2-2 \sin ^{2}\left(\omega_{p} t\right) \sin ^{2}(2|\alpha| t) \\
\operatorname{Tr} \hat{\rho}_{b a_{-}}^{b}(t)=\operatorname{Tr}_{a}\left(\left|\psi_{-}\right\rangle\left\langle\psi_{-}\right|\right)=2+2 \sin ^{2}\left(\omega_{p} t\right) \sin ^{2}(2|\alpha| t)
\end{gathered}
$$

\begin{tabular}{|c|c|c|c|c|}
\hline Time & $\boldsymbol{T r}_{\boldsymbol{b}}\left(\left|\phi_{+}\right\rangle\left\langle\phi_{+}\right|\right)$ & $\boldsymbol{T r}_{\boldsymbol{b}}\left(\left|\phi_{-}\right\rangle\left\langle\phi_{-}\right|\right)$ & $\boldsymbol{T r}_{\boldsymbol{a}}\left(\left|\psi_{+}\right\rangle\left\langle\psi_{+}\right|\right)$ & $\boldsymbol{T r}_{\boldsymbol{a}}\left(\left|\psi_{-}\right\rangle\left\langle\psi_{-}\right|\right)$ \\
\hline$t_{1}=\frac{n \pi}{\omega_{p}}$ & 2 & 2 & 2 & 2 \\
\hline$t_{2}=\left(\frac{1}{2}+n\right) \frac{\pi}{\omega_{p}}$ & 0 & 4 & 0 & 4 \\
\hline
\end{tabular}

The maximum and least traces in $(14 \mathrm{a}-14 \mathrm{~d})$ at time $t_{1}$ and $t_{2}$ are tabulated in table 1.

Table 1. Maximum and least traces of density matrices for Bell states $(14 a-14 d)$ under resonance at time $t_{1}$ and $t_{2}$ where $n=0,1,2 \ldots \ldots$ and $\omega_{p}=2|\alpha|$.

According to CHSH Bell inequality (12), the Bell states (14a) and (14c) under resonance produce weak entanglement. The Bell states (14b) and (14d) produce strong entanglement above the Cirel'son's inequality at the time $t_{2}$.

\subsection{Very Weak Interaction}

\begin{tabular}{|c|c|c|c|c|}
\hline Time & $\boldsymbol{T r}_{\boldsymbol{b}}\left(\left|\phi_{+}\right\rangle\left\langle\phi_{+}\right|\right)$ & $\boldsymbol{T r}_{\boldsymbol{b}}\left(\left|\phi_{-}\right\rangle\left\langle\phi_{-}\right|\right)$ & $\boldsymbol{T r}_{\boldsymbol{a}}\left(\left|\psi_{+}\right\rangle\left\langle\psi_{+}\right|\right)$ & $\boldsymbol{T r}_{\boldsymbol{a}}\left(\left|\psi_{-}\right\rangle\left\langle\psi_{-}\right|\right)$ \\
\hline$t=\frac{n \pi}{2|\alpha| k}$ & 2 & 2 & 2 & 2 \\
\hline$t=\left(\frac{1}{2}+n\right) \frac{\pi}{2|\alpha| k}$ & 0 & 0 & 0 & 0 \\
\hline
\end{tabular}

At very weak interaction

$$
\begin{gathered}
|\alpha|<< \\
\operatorname{Tr}_{b}\left(\left|\phi_{+}\right\rangle\left\langle\phi_{+}\right|\right)=\operatorname{Tr}_{b}\left(\left|\phi_{-}\right\rangle\left\langle\phi_{-}\right|\right)=\operatorname{Tr}_{a}\left(\left|\psi_{+}\right\rangle\left\langle\psi_{+}\right|\right)=\operatorname{Tr}_{a}\left(\left|\psi_{-}\right\rangle\left\langle\psi_{-}\right|\right)=2-2 \sin ^{2}(2|\alpha| k t)
\end{gathered}
$$

The maximum and least traces arising from (16) under very weak interaction are tabulated in table 2.

Table 2. Maximum and least traces of density matrices for Bell states (16) under very weak interaction at time $t_{1}$ and $t_{2}$ where $n=0$, $1,2 \ldots \ldots$.

According to table (2), all the Bell states in (16) under very weak interaction produce weak entanglement.

\subsection{Weak Interaction}

At weak interaction,

$$
|\alpha|<\Delta
$$

and $(7 \mathrm{~d})$ reduces to

$$
\beta=|\alpha| \sqrt{1+k^{2}} \quad ; \quad k>1
$$

Bell state vectors (11a-d) to obtain the trace of the density matrices under weak interaction condition as

$$
\begin{array}{r}
\operatorname{Tr}_{\hat{\rho}_{a b_{+}}^{a}(t)=}=T_{b_{b}}\left(\left|\phi_{+}\right\rangle\left\langle\phi_{+}\right|\right)=2-\frac{8}{k^{2}+1} \sin ^{2}\left(\alpha t \sqrt{1+k^{2}}\right)+\frac{8 \alpha}{\left(k^{2}+1\right)} \sin ^{2}\left(\omega_{p} t\right) \sin ^{2}\left(\alpha t \sqrt{1+k^{2}}\right) \\
-\frac{\left(8 k^{2}+8 \alpha\left(k^{2}+1\right)\right)}{\left(k^{2}+1\right)} \sin ^{2}\left(\omega_{p} t\right) \sin ^{4}\left(\alpha t \sqrt{1+k^{2}}\right)-\frac{\left(2 k^{4}+4 k^{2}-6\right)}{\left(k^{2}+1\right)^{2}} \sin ^{4}\left(\alpha t \sqrt{1+k^{2}}\right. \\
\operatorname{Tr}_{\hat{\rho}_{a b_{-}}^{a}(t)=} \operatorname{Tr}_{b}\left(\left|\phi_{-}\right\rangle\left\langle\phi_{-}\right|\right)=2-\frac{2}{\left(1+k^{2}\right)} \sin ^{2}\left(\omega_{p} t\right) \sin ^{2}\left(2 \alpha t \sqrt{1+k^{2}}\right) \\
+\frac{8 k^{2}}{\left(1+k^{2}\right)^{2}} \sin ^{4}\left(\alpha t \sqrt{1+k^{2}}\right)-\frac{8 k^{2}}{\left(1+k^{2}\right)^{2}} \sin ^{2}\left(\omega_{p} t\right) \sin ^{4}\left(\alpha t \sqrt{1+k^{2}}\right)
\end{array}
$$

We use the condition in (17a) and (17b) in polarization 


$$
\begin{aligned}
& \operatorname{Tr} \hat{p}_{b_{+}}^{b}(t)=T_{r_{a}}\left(\left|\psi_{+}\right\rangle\left\langle\psi_{+}\right|\right)=2-\frac{8}{k^{2}+1} \sin ^{2}\left(\alpha t \sqrt{1+k^{2}}\right)+\frac{8 \alpha}{\left(k^{2}+1\right)} \sin ^{2}\left(\omega_{p} t\right) \sin ^{2}\left(\alpha t \sqrt{1+k^{2}}\right) \\
& -\frac{\left(8 k^{2}+8 \alpha\left(k^{2}+1\right)\right)}{\left(k^{2}+1\right)} \sin ^{2}\left(\omega_{p} t\right) \sin ^{4}\left(\alpha t \sqrt{1+k^{2}}\right)-\frac{\left(2 k^{4}+4 k^{2}-6\right)}{\left(k^{2}+1\right)^{2}} \sin ^{4}\left(\alpha t \sqrt{1+k^{2}}\right.
\end{aligned}
$$

$$
\begin{aligned}
\operatorname{Tr} \hat{\rho}_{b a_{-}}^{b}(t) & =\operatorname{Tr}_{a}\left(\left|\psi_{-}\right\rangle\left\langle\psi_{-}\right|\right)=2-\frac{2}{\left(1+k^{2}\right)} \sin ^{2}\left(\omega_{p} t\right) \sin ^{2}\left(2 \alpha t \sqrt{1+k^{2}}\right) \\
& +\frac{8 k^{2}}{\left(1+k^{2}\right)^{2}} \sin ^{4}\left(\alpha t \sqrt{1+k^{2}}\right)-\frac{8 k^{2}}{\left(1+k^{2}\right)^{2}} \sin ^{2}\left(\omega_{p} t\right) \sin ^{4}\left(\alpha t \sqrt{1+k^{2}}\right)
\end{aligned}
$$

The maximum and least traces corresponding to (18a-d) under weak interaction are given in table 3 .

Table 3. Maximum and least traces of density matrices for Bell states (18a-18d) under weak interaction at time $t_{1}$ and $t_{2}$ where $n=0$, 1 , 2...... and $|\alpha| \sqrt{1+k^{2}}=\omega_{p}$

\begin{tabular}{llll}
\hline Time & $\boldsymbol{T r}_{\boldsymbol{b}}\left(\left|\phi_{+}\right\rangle\left\langle\phi_{+}\right|\right)$ & $\boldsymbol{T r}_{\boldsymbol{b}}\left(\left|\phi_{-}\right\rangle\left\langle\phi_{-}\right|\right)$ & $\boldsymbol{T r}_{\boldsymbol{a}}\left(\left|\psi_{+}\right\rangle\left\langle\psi_{+}\right|\right)$ \\
\hline$t_{1}=\frac{n \pi}{|\alpha| \sqrt{1+k^{2}}}$ & 2 & 4 & 2 \\
$t_{2}=\left(\frac{1}{2}+n\right) \frac{\pi}{2|\alpha| k}$ & $<3$ & $>1$ & $<3$ \\
\hline
\end{tabular}

According to $\mathrm{CHSH}$ Bell inequality, the Bell states (18a) and (18c) under weak interaction produce strong entanglement above the Cirel'son's inequality at the time $t_{2}$. The Bell states (18b) and (18d) produce strong entanglement above the cirel'son's inequality at the time $t_{1}$. The trace reduces as the value of $\mathrm{k}$ increases i.e. $\mathrm{k}>1$.

\subsection{Medium Strength Interaction}

\begin{tabular}{|c|c|c|c|c|}
\hline Time & $\boldsymbol{T r}_{\boldsymbol{b}}\left(\left|\phi_{+}\right\rangle\left\langle\phi_{+}\right|\right)$ & $\boldsymbol{T r}_{\boldsymbol{b}}\left(\left|\phi_{-}\right\rangle\left\langle\phi_{-}\right|\right)$ & $\boldsymbol{T r}_{\boldsymbol{a}}\left(\left|\psi_{+}\right\rangle\left\langle\psi_{+}\right|\right)$ & $\operatorname{Tr}_{a}\left(\left|\psi_{-}\right\rangle\left\langle\psi_{-}\right|\right)$ \\
\hline$t_{1}=\frac{n \pi}{|\alpha| \sqrt{1+k^{2}}}$ & 2 & 4 & 2 & 4 \\
\hline$t_{2}=\left(\frac{1}{2}+n\right) \frac{\pi}{2|\alpha| k}$ & 3 & 0 & 3 & 0 \\
\hline
\end{tabular}

At medium strength interaction,

Table 4. Maximum and least traces of density matrices for Bell states (18a-18d) under medium strength interaction at time $t_{1}$ and $t_{2}$ where $n=0,1,2$.... and $|\alpha| \sqrt{1+k^{2}}=\omega_{p}$

According to table (4), the Bell states in (18a) and (18c) under medium strength interaction produce strong entanglement above the Cirel'son's inequality at the time $t_{2}$. The Bell states (18b) and (18d) produce strong entanglement above the cirel'son's inequality at the time $t_{1}$. The trace reduces as the value of $k$ decreases i.e. $k<1$.

\subsection{Critical (Threshold) Interactions}

$$
|\alpha|=\Delta
$$

and $(7 d)$ becomes $(17 b)$ at $k=1 / 2$. The reduced density matrices take the same format as those presented in equations (18a-d). The maximum and least traces corresponding to $(18 \mathrm{a}-\mathrm{d})$ under critical strength interaction

\begin{tabular}{|c|c|c|c|c|}
\hline Time & $\boldsymbol{T r}_{\boldsymbol{b}}\left(\left|\phi_{+}\right\rangle\left\langle\phi_{+}\right|\right)$ & $\operatorname{Tr}_{b}\left(\left|\phi_{-}\right\rangle\left\langle\phi_{-}\right|\right)$ & $\boldsymbol{T r}_{\boldsymbol{a}}\left(\left|\psi_{+}\right\rangle\left\langle\psi_{+}\right|\right)$ & $\boldsymbol{T r}_{\boldsymbol{a}}\left(\left|\psi_{-}\right\rangle\left\langle\psi_{-}\right|\right)$ \\
\hline$t_{1}=\frac{n \pi}{|\alpha| \sqrt{1+k^{2}}}$ & 2 & 4 & 2 & 4 \\
\hline$t_{2}=\left(\frac{1}{2}+n\right) \frac{\pi}{2|\alpha| k}$ & 2.64 & 0.72 & 2.64 & 0.72 \\
\hline
\end{tabular}
are given in table 5 .

At Critical (threshold) interaction,

Table 5. Maximum and least traces of density matrices for Bell states (18a-d) under critical interaction at time $t_{1}$ and $t_{2}$ where $n=0$, 1 , $2 \ldots . .$. and $|\alpha| \sqrt{1+k^{2}}=\omega_{p}$ 
According to CHSH Bell inequality, the Bell states (18a) and $(18 \mathrm{c})$ under critical interaction produce strong entanglement at the time $\mathrm{t}_{2}$. The Bell states (18b) and (18d) produce strong entanglement above the cirel'son's inequality at the time $\mathrm{t}_{1}$.

\section{Conclusion}

The semi-classical OPO is a good system for demonstration of dynamical evolution of entanglement of polarization states by use of Bell states whose entanglement is tested by use of reduced density matrices in $\mathrm{CHSH}$ Bell inequality.

The CHSH Bell inequality is violated under weak interaction, medium strength and critical interaction for the Bell state vectors (11a) and (11c) hence producing strong entanglement. The traces of density matrices under very weak interaction for all the four Bell state vectors do not violate the $\mathrm{CHSH}$ Bell inequality hence producing weak entanglement which is dynamic in nature.

The CHSH Bell inequality is violated to give a higher trace of $\mathrm{S}=4$ under resonance, weak interaction, medium strength and critical interaction for Bell state vectors (11b) and (11d) hence producing a dynamically stronger entanglement beyond the Cirel'son's inequality of $\mathrm{S}<2 \sqrt{2}$ stated for quantum theory. This presents the OPO as an important tool in quantum optics for possible implementation of quantum key communication protocols in quantum mechanics such as quantum teleportation, quantum key distribution, and dense coding.

\section{References}

[1] Bennett H. and Brassard G. (1993). "Teleporting an unknown quantum state via dual classical and Einstein-Podolsky-Rosen channels". Phys. Rev. Lett. 70, 1895.

[2] Rupert U. (2004). "Quantum teleportation across the Danube". http://www.nature.com/articles/430849a/doi:10.1038/430849a . accessed on 6/7/2014.

[3] Herbst V. and Scheidl F. (2012). "Quantum teleportation over 143 kilometers using active feed-forward". Bibcode: Nature 489.,269M. doi:10.1038/nature 11472 .
[4] Takeda T. (2014). "Deterministic quantum teleportation of photonic quantum bits by a hybrid technique". Nature 500 , 315. DOI: 10. 1038/nature 12366.

[5] Adams W. (2016). China's Quantum Cryptography: Tales from (Quantum) Crypt. National lawreview.http://www.natlawreview.com/article/china-squantum-cryptography-tales-quant-crypt. accessed on $5 / 2 / 2017$

[6] Villar S. and Cassemiro N. (2005). 'Generation of Bright Two-Color Continuous Variable Entanglement," Phys. Rev. Lett. 95, 243603.

[7] Villar S. and Cassemiro N. (2006). Entanglement in the above-threshold optical parametric oscillator. http://arxiv. org/pdf/quant-ph/0610208. accessed on 2/2/2015.

[8] Su X. and Tan A. (2006). ''Experimental demonstration of quantum entanglement between frequency-nondegenerate optical twin beams," Opt. Lett. 31, 1133. DOI:10. 1364/OL.

[9] Shahrokhshahi R. and Pfister O. (2012). Large-scale multipartite entanglement in the quantum optical frequency comb of a depleted-pump optical parametric oscillator. http://arxiv. org/pdf/ 1110. 6450. accessed on 12/6/2015.

[10] Johansson R. (2014). Entangled-state generation and Bell inequality violations in nanomechanical resonators. Phys. Rev. B90, 174307.

[11] Chakrabarti R. and Jenisha J. (2015). Quasi-Bell states in a strongly coupled qubit-oscillator system and their delocalization in the phase space. Phys. Rev. lett 88,148301.

[12] Eberhard P. H. (1999). Violation of Bell's inequality. Phys. Rev. A., 60, RR773.

[13] Akeyo J. O. (2008). Revisiting non-degenerate parametric down-conversion. Pramana-journal of physics Vol. 71, No. 6 pp. 1311-1320.

[14] Ling A. (2008). Entangled state preparation for optical quantum communication. National university of Singapore. http://www.quantumlah.org/media/thesis-alex.pdf.

[15] Munro W. J., Nemoto K. and White A. G. (2001). The Bell inequality: a measure of entanglement. Journal of Modern Optics, Vol. 48, No. 7, 1239-1246.

[16] Preskil J. (1998). Quantum information and computation: Lecture Notes for physics 229. California. http://www. lorentz leidenuniv.ni/quantum computers/preskill/ph. 